\title{
PREVALENCIA DE OTITIS PARASITARIA POR Rhabditis sp. EN EMPRESAS GANADERAS GYR EN CORDOBA, COLOMBIA
}

\section{PREVALENCE OF Rhabditis sp. PARASITIC OTITIS IN CATTLE FARMS GYR IN CORDOBA, COLOMBIA}

\author{
Alfonso Calderón ${ }^{1}$, José Cardona ${ }^{2}$, Marlene Vargas ${ }^{3}$
}

\begin{abstract}
${ }^{1}$ MVZ, M.Sc., Docente, Instituto de Investigaciones Biológicas del Trópico (IIBT), Facultad de Medicina Veterinaria y Zootecnia. Universidad de Córdoba, kilómetro 27 vía Ciénaga de Oro. Estudiante Doctorado en Medicina Tropical, SUE-Caribe, Universidad de Cartagena, e-mail: acalderonr@correo.unicordoba.edu.co; ${ }^{2}$ MVZ, Esp, M.Sc. Ph.D, Docente, Grupo de Investigación en Medicina de Grandes Animales (MEGA), Facultad de Medicina Veterinaria y Zootecnia. Universidad de Córdoba, kilómetro 27 vía Ciénaga de Oro. Correspondencia: e-mail: jacardonaalvarez@correo.unicordoba.edu.co; ${ }^{3}$ MVZ, M.Sc, Ph.D, Docente, Departamento de Medicina Veterinaria. Universidad Federal de Viçosa, Brasil, e-mail: bebel@ufv.br
\end{abstract}

Rev. U.D.C.A Act. \& Div. Cient. 19(1): 149-154, Enero-Junio, 2016

\section{RESUMEN}

Dentro del género Bos indicus, las razas Indubrasil y Gyr son más susceptibles a la otitis parasitaria por Rhabditis bovis. El ganado Gyr, por sus características zootécnicas en el Caribe colombiano, ha sido seleccionado para la producción de leche. El objetivo de la presente investigación fue determinar la prevalencia de otitis parasitaria por Rhabditis sp., en bovinos Gyr, en empresas ganaderas de Córdoba, Colombia. Se implementó un estudio descriptivo prospectivo de conveniencia, donde se recolectaron hisopados de cerumen de los conductos auditivos externos, de 136 bovinos. El criterio de diagnóstico fue la observación directa del movimiento en masa del cerumen y la visualización de los nematodos. La prevalencia de otitis parasitaria fue del $83,82 \%$. Hubo diferencia significativa para las variables otitis clínicas, otorrea y olor. El diagnóstico del parásito demuestra la existencia de Rhabditis sp., como causa de otitis parasitaria en ganados Gyr, en empresas ganaderas de Córdoba.

Palabras clave: Cerumen, diagnóstico, doble propósito, nematodo.

\section{SUMMARY}

Within the genus Bos indicus, the Indubrasil and Gyr breeds are more susceptible to Rhabditis bovis parasitic otitis. The Gyr breed due to its characteristics, in the colombian Caribe has been selected for milk production. The objective was to determine the prevalence of Rhabditis sp., parasitic otitis in Gyr cattle farms in different livestock enterprises in Cordoba, Colombia. A prospective descriptive study was implemented by convenience. Swabs of cerumen from ear canals of 136 cattle were collected in six cattle farms. The diagnostic criterion was the direct observation of the mass movement of cerumen and visualization of nematodes. The prevalence of parasitic otitis was $83.82 \%$. Significant difference was found for the variables clinical otitis, otorrhea and odor, the sex variable was not analyzed. Parasite diagnosis shows the existence of Rhabditis sp. as the cause of parasitic otitis in cattle in livestock farms in Cordoba (Colombia).

Key words: Cerumen, diagnosis, dual purpose, Nematoda

\section{INTRODUCCIÓN}

Las razas de la especie Bos indicus más propensas a la otitis parasitaria por Rhabditis bovis son las Indubrasil y Gyr. Msolla et al. (1993) y Verocai et al. (2007) afirman que esto obedece a la conformación anatómica del oído externo de estas razas y de sus canales auditivos. En países tropicales, la infestación por nematodos del género Rhabditis es la principal causa de la otitis externa en los bovinos; por lo general, se producen brotes y puede afectar hasta el $100 \%$ del hato, como lo referencia Duarte et al. (2001). Leite et al. (2013) afirman que esta problemática representa un problema de salud serio del hato. Vieira et al. (1998) explican que el impacto económico de la otitis en la producción de las empresas ganaderas, se puede presentar por la devaluación de animales genéticamente puros y por los costos de los medicamentos, para prevención y control. Souza et al. (2008) afirman que dentro de las pérdidas económicas, se incluyen la reducción en la producción de leche, la ganancia de peso $y$, en casos extremos, la muerte. 
El género Rhabditis es saprófito, generalmente se desarrolla en materia orgánica en descomposición, como por ejemplo heces frescas; sus miembros son de vida libre, como lo señalan Vieira et al. (2001), Duarte \& Hamdan (2004), Souza et al. (2008). Dentro de las principales fuentes de contagio, se encuentran las actividades grupales como: baños, vacunaciones y pesajes, que por el hacinamiento, favorece la diseminación rápida del parásito, a partir de animales enfermos a sanos (Msolla et al. 1986; Cardona et al. 2010).

Ushewokunze et al. (1999) señalaron que un factor de riesgo es la presencia de moscas; Obatolu et al. (1999), en África, mencionan que la mosca doméstica podría ser un vector de nematodos, igualmente Verocai et al. (2007), en Brasil, sugirieren que la mosca doméstica podría tener un papel importante en la diseminación del nematodo; sin embargo, Leite et al. (2012) postulan que aún hace faltan estudios, que clarifiquen el mecanismo de trasmisión.

Abdalla et al. (2008) y Cardona et al. (2010) sostienen que la otitis parasitaria por Rhabditis produce estrés y dolor, debido al proceso inflamatorio que causa el estrechamiento del canal auditivo y la pérdida de audición.

Dentro de los signos clínicos, Msolla et al. (1993) y Verocai et al. (2007) incluyen secreción purulenta y fétida, otorrea que predispone a la miasis y signos nerviosos, como hemiplejía facial, que afecta la aprehensión y la masticación, que reducen drásticamente la producción y la ganancia de peso. Abdalla et al. (2008) y Souza et al. (2008) manifiestan que la pérdida del equilibrio y de la coordinación, se presenta por el síndrome vestibular, caracterizado por la inclinación de la cabeza, constante nistagmo de tipo horizontal y caída de orejas, párpados, nariz y los labios se tornan flácidos.

El objetivo de este estudio fue determinar la prevalencia de otitis parasitaria por Rhabditis sp., en bovinos Gyr, en empresas ganaderas doble propósito, en cuatro municipios del departamento de Córdoba, Colombia.

\section{MATERIALES Y MÉTODOS}

Se implementó un estudio descriptivo, que involucró zonas rurales de los municipios de Planeta Rica, Cereté, San Carlos y Sahagún, de la región del Medio Sinú, del departamento de Córdoba, donde las características geoclimáticas son similares. Córdoba está ubicado entre los coordenadas $7^{\circ} 23^{\prime}$ y $9^{\circ} 26^{\prime}$ de latitud Norte y los 7452' y 76032' de longitud al Oeste del meridiano de Greenwich, a una altura de $30 \mathrm{msnm}$, con temperatura promedio anual de $28^{\circ} \mathrm{C}$, humedad relativa del $82 \%$, precipitación media anual de $1400 \mathrm{~mm}$; pertenece a la formación climática de bosque tropical lluvioso y presentan dos épocas de máxima y mínima precipitación, bien definidas (Palencia et al. 2006).
Para calcular el tamaño de la muestra, se utilizó el censo de la raza Gyr, del registro de la Asociación Colombiana de Criadores de Ganado Cebú del 2010, que fue de 2.728 bovinos puros (Fedegan \& Ganacor, 2013) y se estableció, como tamaño de la muestra, 136 bovinos puros de la raza Gyr, con un intervalo de confianza del $95 \%$, un margen de error del 0,05 y un prevalencia del 90\% (Casal \& Mateu, 2003). Esta muestra, se tomó en las cuatro mayores empresas ganaderas de la raza Gyr, empresas en las que la población fluctuó alrededor de 300 ejemplares. De cada hato se tomaron 36 muestras, tratando que la población de adultos y jóvenes fuera igual y pertenecía a ambos sexos.

Todos los bovinos involucrados, en el presente estudio, se inmovilizaron en un brete y se hizo sujeción de la cabeza, para garantizar la integridad física del personal involucrado y hacer más fácil la recolección del cerumen de los conductos auditivos externos. El cerumen, se obtuvo mediante el hisopado de los conductos auditivos externos, observando en forma directa el movimiento en masa del cerumen. Posteriormente, el material recolectado se depositó en tubos de ensayo, debidamente identificados y luego transportados a temperatura ambiente al Laboratorio de Parasitología de la Facultad de Medicina Veterinaria de la Universidad de Córdoba, donde se observó utilizando un microscopio estereoscopio. La observación en forma directa del movimiento en masa del cerumen y la visualización de los nematodos fue el criterio de diagnóstico. El estudio de campo, se realizó entre julio y noviembre de 2013.

Además se realizó la evaluación de la condición corporal (CC) de cada animal, cuyo objetivo fue la estimación de las reservas corporales, grasa y músculos, mediante la calificación visual y la palpación de las costillas, la columna vertebral, los huesos de la cadera e inserción de la cola. En esta evaluación se compara lo observado con un modelo, al que se le han predeterminado unos valores numéricos. Uno corresponde a un animal emaciado y nueve a uno obeso (Houghton et al. 1990; Wagner et al. 1988: Kabaleski, 2013).

La identificación de los signos clínicos de otitis parasitaria por Rhabditis sp., se realizó por medio de un examen clínico, en donde a la palpación en las bases de las orejas, se evaluó la presencia de dolor y linfadenitis de los nódulos linfáticos mandibulares. Este examen, se hizo en ambos conductos auditivos. Las valoraciones siempre fueron realizadas por el mismo Médico Veterinario Zootecnista (MVZ), quien es uno de los autores.

Los datos, se organizaron en tablas y se analizaron en forma descriptiva en el Software SAS. Se usó Chi.cuadrado, para ver si los datos obtenidos presentan variaciones estadísticamente significativas (Gómez et al. 2003). 
El estudio fue considerado de bajo riesgo por el Comité de Ética del Instituto de Investigaciones Biológicas del Trópico (IIBT), de la Facultad de Medicina Veterinaria y Zootecnia, de la Universidad de Córdoba. La manipulación de los bovinos, para la toma de las muestras, se realizó bajo la supervisión de un MVZ, quien siempre tuvo en cuenta los procedimientos de toma de muestras, manejo y conservación, según las normas de las buenas prácticas de laboratorio y las normas éticas, técnicas y científicas, conforme a la Ley 84 (Congreso de Colombia, 1989). A lo largo del estudio, se mantuvo la confidencialidad de la información de las fincas y de los animales evaluados. Para evitar errores de apreciación, la valoración clínica de la otorrea siempre fue realizada por el mismo profesional.

\section{RESULTADOS Y DISCUSIÓN}

De los 136 ejemplares de bovinos de la raza Gyr, donde el $97,8 \%$ fueron hembras y el $2,2 \%$, machos (Tabla 1 ), provenientes de las cuatro empresas ganaderas, dedicadas a su cría y fomento, ubicadas en áreas rurales de Córdoba, se obtuvo una prevalencia del $83,82 \%$, para otitis clínica parasitaria por Rhabditis sp.

En Colombia, no se han realizado estudios de prevalencia de otitis parasitaria clínica por Rhabditis sp. En ganado Gyr, sólo se han reportado dos estudios de frecuencia en Córdoba (Cardona et al. 2012), donde reporta una frecuencia de $63,2 \%$ y Cardona et al. (2014), en Sucre, del 85,6\%. Este tipo de otitis parasitaria se presenta, principalmente, en ganados Gyr, por la disposición del oído externo y sus conductos auditivos, el acúmulo del cerumen, como lo reportan Msolla et al. (1993) y Verocai et al. (2007). Además influyen las condiciones de humedad y de calor de la zona, escenarios que ofrecen condiciones favorables, para el desarrollo del nematodo Rhabditis spp. (Verocai et al. 2007).

En países tropicales, como Tanzania (Msolla et al. 1993), Kenia (Round, 1962; Matandala et al. 2002), Zimbabue (Ushewokunze et al. 1999) y Brasil (Vieira et al. 1998), la infestación por nematodos del género Rhabditis es la principal causa de la otitis externa, en el ganado bovino.

Vieira et al. (1998), en Brasil, en empresas ganaderas de Gyr puro, observó un alto índice de prevalencia de otitis parasitaria por Rhabditis sp; Leite et al. (1993), en Minas Gerais, del 93\%; Vieira et al. (1998), en Goiás, del 78,43\%; Duarte et al. (2001), en Minas Gerais, del 60,1\%; Verocai et al. (2007), en Río de Janeiro, del 90,9\% y Leite et al. (2013), en Minas Gerais, del $52,2 \%$. Estos reportes están demostrando un alto potencial de trasmisión y la necesidad de implementar me-

Tabla 1. Prevalencia de otitis parasitaria clínica por Rhabditis sp., en bovinos Gyr, en cuatro empresas ganaderas de Córdoba, por sexo.

\begin{tabular}{|l|c|c|c|c|c|c|}
\hline \multirow{2}{*}{ Categoría } & \multicolumn{2}{|c|}{ Negativos } & \multicolumn{2}{c|}{ Positivos } & \multicolumn{2}{c|}{ TOTAL } \\
\cline { 2 - 7 } & $\mathbf{\%}$ & $\mathbf{n}$ & $\mathbf{\%}$ & $\mathbf{n}$ & $\mathbf{1}$ & N \\
\hline Hembras & 13,97 & 19 & 83,8 & 114 & 97,8 & 133 \\
\hline Machos & 2,20 & 3 & 0,00 & 0 & 2,20 & 3 \\
\hline TOTAL & 16,2 & 22 & 83,8 & 114 & 100 & 136 \\
\hline
\end{tabular}

didas de control. Msolla et al. (1993), Vieira et al. (2001) y Duarte \& Hamdan (2004) afirman que esta parasitosis puede causar disminución de la producción de leche y disminución de la ganancia de peso, ocasionando así pérdidas económicas para los productores.

El 72,78\% de la población evaluada correspondió a bovinos adultos, mientras que el $27,20 \%$, a bovinos jóvenes y la prevalencia, de acuerdo al grupo etario edad (adulto y joven), se presenta en la tabla 2. La mayor prevalencia, se reveló dentro del grupo adulto $(64,70 \%)$, por ser la mayor población evaluada, pero se observa que bovinos jóvenes, también se están infectando. Estos resultados concuerdan con el estudio de Martins et al. (1971) y Duarte et al. (2001), quienes asociaron síntomas de otitis a ganado adulto, mientras que en los animales jóvenes, las infecciones observadas fueron generalmente pocas.
Diversos estudios han demostrado que existe gran diferencia en las manifestaciones clínicas de la enfermedad, entre animales adultos y jóvenes. Martins et al. (1971) afirmaron que las infecciones en adultos se asociaron, generalmente, con síntomas de otitis, mientras infecciones en los terneros son siempre leves y asintomáticas. Leite et al. (1993) y Duarte et al. (2001) concluyen que en vacas Gyr la prevalencia de otitis parasitaria fue significativamente mayor por la presencia de cuernos y puede ser considerado como un factor de riesgo, ya que los cuernos grandes pueden comprimir el canal auditivo y crear un ambiente más propicio, para un mayor parasitismo.

Se determinó que existe asociación entre otitis parasitaria y las variables otorrea, otitis clínica y olor fétido $(p<0,001)$; para estos casos, la otorrea, la otitis clínica y el olor fétido están asociados con la otitis parasitaria. En contraste, no se 
Tabla 2. Presencia de otitis parasitaria clínica por Rhabditis sp., en bovinos Gyr, en cuatro empresas ganaderas de Córdoba, por edad.

\begin{tabular}{|l|c|c|c|c|c|c|}
\hline \multirow{2}{*}{ Categoría } & \multicolumn{2}{|c|}{ Adulto } & \multicolumn{2}{c|}{ Joven } & \multicolumn{2}{c|}{ TOTAL } \\
\cline { 2 - 7 } & $\mathbf{\%}$ & $\mathbf{n}$ & $\mathbf{\%}$ & $\mathbf{n}$ & $\mathbf{\%}$ & $\mathbf{N}$ \\
\hline Negativo & 8,1 & 11 & 8,1 & 11 & 16,2 & 22 \\
\hline Positivo & 64,7 & 88 & 19,12 & 26 & 83,8 & 114 \\
\hline TOTAL & 72,8 & 99 & 27,2 & 37 & 100 & 136 \\
\hline
\end{tabular}

encontró asociación para la variable condición corporal con otitis parasitaria. Florio et al. (2012), respecto a la presentación de otitis parasitaria y la condición corporal, concluyeron que la condición corporal depende de otros factores, como la nutrición, el estado fisiológico del animal, entre otros.

En la tabla 3, se presenta la estadística descriptiva de la otitis, donde se observó que el $77,21 \%$ fue otitis clínica y el $6,61 \%$ no fue clínica. Esto se determinó por la no evidencia de signos clínicos, pero sí se observó en el cerumen, el movimiento en masa y presencia de nematodos, parámetros de diagnósticos considerados como positivos para otitis parasitaria, en este estudio.

Con relación al grado de la otorrea o salida de flujo o secreciones procedentes del conducto auditivo eterno (Tabla 4 ), en el $82,35 \%$ fueron evidentes y en el $1,47 \%$, sin otorrea, pero con diagnóstico positivo, a partir del cerumen. Esto coincide con estudios previos, donde se describe que animales con otitis parasitaria, en la mayoría de los casos, presentan la afección en ambas orejas, acompañadas principalmente de otorrea con secreción, algunas veces purulenta, fétida y proceso inflamatorio, que causa el estrechamiento del canal auditivo, dolor a la palpación en las bases de las orejas y linfadenitis de los nódulos linfáticos mandibulares (Vieira et al. 1998; 2001; Abdalla et al. 2008).

El olor fétido, se manifestó en el 68,38\% y, en el 31,54\%, el olor fue normal, pero en el 15,44\%, el diagnóstico fue positivo, a partir del cerumen, ya que se demostró movimiento en masa del cerumen y presencia de nematodos; en el $16,17 \%$, el olor fue normal y no se evidenciaron estos parámetros, considerados como positivos para otitis parasitaria, en este estudio (Tabla 5).

Las muestras fueron colectadas en los meses de mayor precipitación, por lo que se puede inferir, que la alta prevalencia puede estar influenciada por la presentación de lluvias y la elevada humedad relativa. Vieira et al. (2001) y Duarte \& Hamdan (2004) afirmaron que estas condiciones favorecen una mayor presentación de material en descomposición, que mejora la condición del ciclo reproductivo del nematodo. Igualmente, Leite et al. (2013) determinaron que la alta humedad relativa y la elevada temperatura ambiental favoreció la transmisión de Rhabditis sp., agente etiológico de la otitis parasitaria.

Tabla 3. Presencia o ausencia de otitis clínica en bovinos Gyr, en cuatro empresas ganaderas de Córdoba ( $<<0,001)$.

\begin{tabular}{|l|c|c|c|c|c|c|}
\hline \multirow{2}{*}{ Categoría } & \multicolumn{2}{|c|}{ Sin otitis clínica } & \multicolumn{2}{c|}{ Con otitis clínica } & \multicolumn{2}{c|}{ TOTAL } \\
\cline { 2 - 7 } & $\mathbf{\%}$ & $\mathbf{n}$ & $\mathbf{\%}$ & $\mathbf{n}$ & $\mathbf{\%}$ & $\mathbf{N}$ \\
\hline Negativo & 16,2 & 22 & 0,00 & 0 & 16,2 & 22 \\
\hline Positivo & 6,6 & 9 & 77,2 & 105 & 83,8 & 114 \\
\hline TOTAL & 22,8 & 31 & 77,2 & 105 & 100 & 136 \\
\hline
\end{tabular}

Tabla 4. Presencia o ausencia de otorrea en bovinos Gyr, en empresas ganaderas de Córdoba $(\mathrm{p}<0,001)$.

\begin{tabular}{|l|c|c|c|c|c|c|}
\hline \multirow{2}{*}{ Categoría } & \multicolumn{2}{|c|}{ Sin otorrea } & \multicolumn{2}{c|}{ Con otorrea } & \multicolumn{2}{c|}{ TOTAL } \\
\cline { 2 - 7 } & $\mathbf{\%}$ & $\mathbf{n}$ & $\mathbf{\%}$ & $\mathbf{n}$ & $\mathbf{\%}$ & $\mathbf{N}$ \\
\hline Negativo & 16,2 & 22 & 0,00 & 0 & 16,2 & 22 \\
\hline Positivo & 1,4 & 2 & 82,4 & 112 & 83,8 & 114 \\
\hline TOTAL & 17,6 & 24 & 82,4 & 112 & 100 & 136 \\
\hline
\end{tabular}


Tabla 5. Caracterización del olor en bovinos Gyr, en empresas ganaderas de Córdoba $(p<0,001)$.

\begin{tabular}{|l|c|c|c|c|c|c|}
\hline \multirow{2}{*}{ Categoría } & \multicolumn{2}{|c|}{ Fétido } & \multicolumn{2}{c|}{ Normal } & \multicolumn{2}{c|}{ TOTAL } \\
\cline { 2 - 7 } & $\mathbf{\%}$ & $\mathbf{n}$ & $\mathbf{\%}$ & $\mathbf{n}$ & $\mathbf{\%}$ & $\mathbf{N}$ \\
\hline Negativo & 0,00 & 0 & 16,2 & 22 & 16,2 & 22 \\
\hline Positivo & 68,4 & 93 & 15,4 & 21 & 83,8 & 114 \\
\hline TOTAL & 68,4 & 93 & 31,6 & 43 & 100 & 136 \\
\hline
\end{tabular}

La prevalencia de otitis parasitaria clínica por Rhabditis sp., en ganados Gyr, en empresas ganaderas de Córdoba fue alta y es necesario establecer un adecuado manejo de toda la materia en descomposición, existente en lugares donde se produce una mayor concentración de animales, como corrales y bretes. Estas medidas deben realizarse con el fin de erradicar dichos focos de infección e implementar medidas de control integrado, que incluyan estrategias químicas, biológicas o culturales e implementar planes sanitarios curativos y profilácticos.

Agradecimientos: Los autores agradecen a todos los ganaderos y asistentes técnicos, quienes permitieron la toma de las muestras en sus empresas ganaderas. Conflictos de intereses: Este manuscrito fue preparado y revisado con la participación de todos los autores, quienes declaramos que no existe conflicto de intereses que pongan en riesgo la validez de los resultados presentados.

\section{BIBLIOGRAFÍA}

1. ABDALLA, M.S.; PEIXOTO, T.C.; ALVES P., A.M.; FRANÇA, T.N.; BRITO, M.F. 2008. Aspectos anatómopatológicos de la otitis causada por Rhabditis sp. en bovinos en Río de Janeiro. Brasil. Disponible desde internet en: http://www.sovergs.com.br/conbravet2008/anais/cd/resumos/r0743-1.pdf (con acceso 14/04/2015).

2. CARDONA, J.; GONZÁLEZ, M.; ÁLVAREZ, J. 2010. Otitis bovina por Rhabditis bovis en Córdoba, Colombia. Reporte de dos casos. Rev. MVZ Córdoba (Colombia) 15:2240-2244.

3. CARDONA, J.; GONZÁLEZ, M.; ÁLVAREZ, J. 2012. Frecuencia de otitis parasitaria clínica por nematodos Rhabditiformes (Rhabditis spp.) en seis fincas de la raza Gyr en Córdoba, Colombia. Rev. Col. Cienc Pec. 25:417-421.

4. CARDONA, J.; CALDERÓN, A.; PERDOMO, S. 2014. Frecuencia de otitis parasitaria bovina (Rhabditis spp.) en tres explotaciones de la raza Gyr en el Departamento de Sucre, Colombia. Rev. Científica, FCV-LUZ (Venezuela) 24(1):22-26.
5. CASAL, J.; MATEU, E. 2003. Tipos de muestreo. Rev. Epidem. Med. Prev (España).1: 3-7.

6. CONGRESO DE COLOMBIA. 1989. Estatuto nacional de protección animal. Ley 84/1989 del 27 de Diciembre. Diario Oficial 39120 de Diciembre 27 de 1989. Disponible desde Internet en: http://spac-05. tripod.com/id24.html (con acceso 14/04/2015).

7. DUARTE, E.; MELO, M.; HAMDAN, J. 2001. Epidemiological aspects of bovine parasitic otitis caused by Rhabditis spp. and/or Raillietia spp. In the state of Minas Gerais, Brazil. Vet. Parasitol (Brasil). 101(1):45-52.

8. DUARTE, E.; HAMDAN, J.S. 2004. Otitis in cattle, an aetiological review. J. Vet. Med. B Infect Dis Vet Public Health (Alemania). 51(1):1-7.

9. FEDERACIÓN COLOMBIANA DE GANADEROS FEDEGAN-; FEDERACIÓN GANADERA DE CORDOBA -GANACOR-. 2013. Informe final ciclo II 2013. Coordinación regional Córdoba (Colombia). $15 p$.

10. FLORIO, L.J.; TAMASAUKAS, R.; RIVERA, S. 2012. Diagnóstico participativo de hemotrópicos en bovinos a nivel de pequeños productores y productoras de ganadería doble propósito en el sur del estado Aragua en la República Bolivariana de Venezuela. AICA (España). 2:163-170.

11. GÓMEZ, G.M.; DANGLOT, B.D.; LEOPOLDO VEJA, F.L. 2003. Sinopsis de pruebas estadísticas nas paramétricas. Cuándo usarlas. Rev Mex Pediatr 70(2): 91-99. 2003.

12. HOUGHTON, P.L.; LEMENAGER, R.P.; HORSTMAN, L.A.; HENDRIX, K.S.; MOSS, G.E. 1990. Effects of body composition, pre- and postpartum energy level and early weaning on reproductive performance of beef cows and preweaning calf gain. J. Anim Sci 68(5):1438-1446.

13. KABALESKI, C.D. 2013. Condición corporal en ganado de carne. Disponible en: http://www.produccion- 
animal.com.ar/informacion_tecnica/cria_condicion corporal/50-Condicion_Corporal_Carne.pdf (con acceso 14/04/2015).

14. LEITE, R.C.; NUNES, V.A.; NUNES, I.J.; COSTA, A.L.; FACCINI, J.L.H.; LOPES, C.W.G. 1993. Otite parasitária bovina por nematóides rhabditiformes: aspectos epidemiológicos e clínicos. Rev. Bras. Med. Vet. 5(2):49-51.

15. LEITE, P.V.B.; CUNHA, L.M.; OLIVEIRA, P.R.; LEITE, L.B.; LEITE, R.C. 2012. Farmers' perception about parasitic otitis in Gyr breed from three states of Brazil. Pesq. Vet. Bras. 32(9):855-858.

16. LEITE, P.V.B.; LEITE, L.B.; CUNHA, A.P. da SILVA, M.X.; BELLO, A.C.P.P.; DOMINGUES, L.N.; LEITE, A.; LEITE, R.C. 2013. Clinical aspects and dynamics of auricular parasitosis in Gir cattle. Pesq. Vet. Bras. 33(3):319-325.

17. MARTINS, J.R.; NUNES, I.J.; RIBEIRAL, L.A.; ROSAZ, C.E.E.; NUNES, V.A. 1971. Nota sobre a ocorrência de Rhabditidae (Nematoda, Rhabditida) relacionados com otite em bovinos na região geo-econômica de Brasília, DF. Ciência e Cultura. 23:248-249.

18. MATANDALA, M.; MUGERA, G.; NGATIA, T. 2002. Prevalence of bovine (nematodes) otitis in Kenya. Kenya Vet. 25:32-35.

19. MSOLLA, P.; MATAFU, E.; MONRAD, J. 1986. Epidemiology of bovine parasitic otitis in Tanzania. Trop. Anim. Health Prod. (Estados Unidos). 18:51-52.

20. MSOLLA, P.; SEMUGURUKA, W.D.; KASSUKU, A.A.; SHOO, M.K. 1993. Clinical observations on bovine parasitic otitis in Tanzania. Trop. Anim. Health Prod. 25(1):15-18.

21. OBATOLU, U.U.; PFUKENYI, D.M.; USHE, T. 1999. A retrospective epidemiological study of parasitic otitis in cattle in the South-East Lowveld of Zimbabwe. Zimbabwe Vet. J. 30(1):19-24.

22. PALENCIA, G.; MERCADO, T.; COMBATT, E. 2006. Estudio agroclimatológico del departamento de Córdoba. Universidad de Córdoba. Editorial Gráficas del Caribe.129p.
23. ROUND, M.C. 1962. The helminth parasites of domesticated animals in Kenya. J. Helminthol. (Inglaterra). 36(4):375-449.

24. SOUZA, W.A.; CALDERARO, T.; MATOSINHO, R.O.; PRATELLESI, N.B.; SOLIVA, A.N.; NEVES, M.F. 2008. Otite parasitária causada por nematoides Rhabditiformes. Rev. Cient. Eletônica Med. Vet. 6(11):1-5. Disponible desde Internet en: http://www. faef.revista.inf.br/imagens arquivos/arquivos destaque/SXkBRrCphpOGdSJ_2013-6-13-15-43-8.pdf (con acceso 14/04/2015).

25. STATISTICAL ANALYSIS SYSTEMS -SAS-. 2001. User's Guide (Version 9.1), Cary (USA). Institute. SAS/STAT.

26. USHEWOKUNZE, O.U.; PFUKENYI, D.; USHE, T. 1999. A retrospective epidemiological study of parasitic otitis in cattle in South-East Lowveld of Zimbabwe. Zimbabwe Vet. J. 30(1):19-24.

27. VEROCAI, G.G.; FERNANDES, J.I.; CORREIA, T.R.; MELO, R.M.P.S.; ALVES, P.A.M.; SCOTT, F.B. 2007. Otite parasitária bovina por nematóides rhabditiformes em vacas Gir no Estado do Rio de Janeiro. Brazil J. Parasitol. Vet. 16(2):105-107.

28. VIEIRA, M.; Da SILVA, L.; BORGES, N.; BARROS, J.; IGLESIAS, A.; VIEIRA, E. 1998. Estudo da prevalíncia de otites clìnicas por Rhabidtis $s p$. em bovinos da raça Gir no estado de Goiás. Anais Esc. Agron. Vet. (Brasil) 28:19-29.

29. VIEIRA, M.; Da SILVA, L.; ARAÚJO, J.; ANDRADE, M.; FIORAVANTI, M.; SILVA E. 2001. Otites parasitárias por nematóides rhabditiformes em bovinos: avaliação de tratamentos. Ciênc. Anim. Bras. 2(1):5155.

30. WAGNER, J.J.; LUSBY, K.S.; OLTJEN, J.W.; RAKESTRAW, J.; WETTEMANN R.P.; WALTERS, L.E. 1988. Carcass composition in mature Hereford cows: estimation and effect on daily metabolizable energy requirement during winter. J. Anim Sci 66(3): 603-612.

Recibido: Abril 20 de 2015

Aceptado Marzo 29 de 2016

Cómo citar:

Calderón, A.; Cardona, J.; Vargas, M. 2016. Prevalencia de otitis parasitaria por Rhabditis sp. en empresas ganaderas Gyr en Córdoba, Colombia. Rev. U.D.C.A Act. \& Div. Cient. 19(1): 149-154. 